PROCEEDINGS OF THE

AMERICAN MATHEMATICAL SOCIETY

Volume 127, Number 7, Pages 1903-1915

S 0002-9939(99)04671-7

Article electronically published on March 3, 1999

\title{
CONGRUENCE-PRESERVING EXTENSIONS OF FINITE LATTICES TO SECTIONALLY COMPLEMENTED LATTICES
}

\author{
G. GRÄTZER AND E. T. SCHMIDT \\ (Communicated by Lance W. Small)
}

\begin{abstract}
In 1962, the authors proved that every finite distributive lattice can be represented as the congruence lattice of a finite sectionally complemented lattice. In 1992, M. Tischendorf verified that every finite lattice has a congruence-preserving extension to an atomistic lattice.

In this paper, we bring these two results together. We prove that $e v$ ery finite lattice has a congruence-preserving extension to a finite sectionally complemented lattice.
\end{abstract}

\section{INTRODUCTION}

Early results on related structures (the automorphism group, the congruence lattice, and so on) of a lattice were characterization theorems, typified by the following result of R. P. Dilworth:

Theorem 1. Let $D$ be a finite distributive lattice. Then there exists a finite lattice $L$ such that the congruence lattice of $L$ is isomorphic to $D$.

A stronger form of this result was published in G. Grätzer and E. T. Schmidt [6]:

Theorem 2. Every finite distributive lattice $D$ can be represented as the congruence lattice of a finite sectionally complemented lattice $L$.

In the last decade, the emphasis has shifted from representation theorems to extension theorems, typified by the following important result of M. Tischendorf [12]:

Theorem 3. Every finite lattice has a congruence-preserving embedding to a finite atomistic lattice.

In this paper, in the spirit of Tischendorf's result, we shall prove the extension form of Theorem 2:

Received by the editors July 16, 1996 and, in revised form, September 22, 1997.

1991 Mathematics Subject Classification. Primary 06B10; Secondary 08A05.

Key words and phrases. Congruence lattice, congruence-preserving embedding, sectionally complemented lattice, finite.

The research of the first author was supported by the NSERC of Canada.

The research of the second author was supported by the Hungarian National Foundation for Scientific Research, under Grant No. T7442.

(C)1999 American Mathematical Society 
Theorem. Every finite lattice $K$ has a congruence-preserving embedding into a finite sectionally complemented lattice $L$.

This result does not hold for infinite lattices: $F\left(\aleph_{2}\right)$ (the free lattice on $\aleph_{2}$ generators) does not have a congruence-preserving embedding into a sectionally complemented lattice according to M. Ploščica, J. Tůma, and F. Wehrung [10]. The problem is open for lattices of size $\aleph_{0}$ and $\aleph_{1}$.

See G. Grätzer and E. T. Schmidt [8] for a discussion of characterization theorems vs. extension theorems, and see G. Grätzer, H. Lakser, and E. T. Schmidt [2], [3], [4], and G. Grätzer and E. T. Schmidt [8] for further examples of extension theorems.

\section{Preliminaries}

We use the basic concepts and notations as in [1].

A finite lattice $L$ is atomistic if every element is a join of atoms; $L$ is sectionally complemented if, for every $a \leq b$ in $L$, there is an element $c$ that is a complement of $a$ in the interval $[0, b]$. Obviously, every finite sectionally complemented lattice is atomistic, but not conversely; the seven element lattice $K$ of Figure 1 witnesses this.

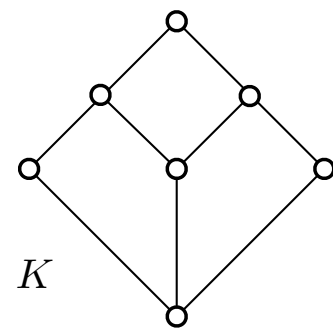

Figure 1

Let $L$ be a lattice. If $K$ is a sublattice of $L$, we call $L$ an extension of $K$. If $L$ is an extension of $K, \Theta$ is a congruence of $K$, and $\Phi$ is a congruence of $L$, then $\Phi$ is an extension of $\Theta$ to $L$ iff the restriction of $\Phi$ to $K$ equals $\Theta$. We say that $K$ in $L$ has the Congruence Extension Property iff every congruence of $K$ has an extension to $L$.

We call $L$ a congruence-preserving extension of $K$ iff every congruence of $K$ has exactly one extension to $L$. In this case, the congruence lattice of $K$ is isomorphic to the congruence lattice of $L$; in formula, Con $K \cong$ Con $L$.

Let $M$ be a finite poset such that $\inf \{a, b\}$ exists in $M$, for all $a, b \in M$. We define in $M$ :

$$
\begin{array}{ll}
a \wedge b=\inf \{a, b\}, & \text { for all } a, b \in M ; \\
a \vee b=\sup \{a, b\}, & \text { whenever } \sup \{a, b\} \text { exists. }
\end{array}
$$

This makes $M$ into a finite chopped lattice. Note that if $L$ is a finite lattice with unit, 1 , then $L-\{1\}$ is a finite chopped lattice; and conversely, if $M$ is a finite chopped lattice, then $L=M \cup\{1\}$ is a finite lattice from which we obtain $M$ by chopping off 1. 
An equivalence relation $\Theta$ on the chopped lattice $M$ is a congruence relation iff, for all $a_{0}, a_{1}, b_{0}, b_{1} \in M$,

$$
\begin{aligned}
& a_{0} \equiv b_{0}(\Theta), \\
& a_{1} \equiv b_{1}(\Theta),
\end{aligned}
$$

imply that

$$
\begin{aligned}
& a_{0} \wedge a_{1} \equiv b_{0} \wedge b_{1}(\Theta), \\
& a_{0} \vee a_{1} \equiv b_{0} \vee b_{1}(\Theta), \quad \text { whenever } a_{0} \vee a_{1} \text { and } b_{0} \vee b_{1} \text { both exist. }
\end{aligned}
$$

The set Con $M$ of all congruence relations of $M$ is a lattice.

An ideal $I$ of a finite chopped lattice $M$ is a non-empty subset $I \subseteq M$ with the following two properties:

(i) $i \wedge a \in I$, for $i \in I$ and $a \in M$;

(ii) $i \vee j \in I$, for $i, j \in I$, provided that $i \vee j$ exists in $M$.

The ideals of the finite chopped lattice $M$ form the finite lattice Id $M$.

The following lemma was published in G. Grätzer [1].

Lemma 4 (G. Grätzer and H. Lakser). Let $M$ be a finite chopped lattice. Then for every congruence relation $\Theta$ of $M$, there exists exactly one congruence relation $\bar{\Theta}$ of Id $M$ such that, for $a, b \in M$,

$$
(a] \equiv(b](\bar{\Theta}) \quad \text { iff } \quad a \equiv b(\Theta) .
$$

In particular, $\operatorname{Con} M \cong \operatorname{Con}(\operatorname{Id} M)$.

In the proof of the Theorem, we need a more detailed form of Theorem 2.

Theorem $\mathbf{2}^{\prime}$. For every finite distributive lattice $D$, there is a lattice $L$ with the following properties:

(i) $L$ is finite;

(ii) $L$ is sectionally complemented;

(iii) Con $L$ is isomorphic to $D$;

(iv) there is an independent set of atoms

$$
Q=\left\{q_{\Phi} \mid \Phi \in \mathrm{J}(\operatorname{Con} L)\right\}
$$

of $L$ such that $\Phi=\Theta\left(0, q_{\Phi}\right)$, for $\Phi \in \mathrm{J}(\operatorname{Con} L)$, and there is a one-to-one correspondence between congruences and certain subsets of $Q$; the subset that corresponds to the congruence $\Theta$ of $L$ is

$$
\left\{q_{\Phi} \mid \Phi \in \mathrm{J}(\operatorname{Con} L) \text { and } \Phi \leq \Theta\right\} .
$$

(Recall that a set of atoms is independent, if they generate a Boolean sublattice; $\mathrm{J}(\operatorname{Con} L)$ is the set of join-irreducible congruences of $L$.)

The two standards proofs of Theorem 2 (the original proof in [6] and the proof, due to the first author and H. Lakser, published in [1]) both exhibit the set $Q$ satisfying (iv).

\section{Sectionally complemented lattices}

In this section, we present a new construction of finite sectionally complemented lattices. 
Theorem 5. Let $A$ and $B$ be (disjoint) finite sectionally complemented lattices with zero elements $0_{A}$ and $0_{B}$, respectively. Let $p_{A}$ be an atom of $A$ and let $p_{B}$ be an atom of $B$.

Form the partial lattice $M$ by identifying $0_{A}$ with $0_{B}$ and $p_{A}$ with $p_{B}$. Then $M$ is a finite chopped lattice and $\operatorname{Id} M$ is a finite sectionally complemented lattice.

Proof. Let $0=0_{A}=0_{B}$ and $p=p_{A}=p_{B}$. Obviously, $M$ is a finite chopped lattice.

An ideal $I$ of $M$ can be represented by a pair of elements $\left\langle i_{A}, i_{B}\right\rangle$ such that $i_{A} \in A, i_{B} \in B, I \cap A=\left(i_{A}\right], I \cap B=\left(i_{B}\right]$, and $i_{A} \wedge p=i_{B} \wedge p$.

To show that Id $M$ is sectionally complemented, let $I \subseteq J$ be two ideals of $M$, represented by $\left\langle i_{A}, i_{B}\right\rangle$ and $\left\langle j_{A}, j_{B}\right\rangle$, respectively. Let $s_{A}$ be the sectional complement of $i_{A}$ in $j_{A}$ and let $s_{B}$ be the sectional complement of $i_{B}$ in $j_{B}$. If

$$
p \wedge s_{A}=p \wedge s_{B}
$$

then $\left\langle s_{A}, s_{B}\right\rangle$ is an ideal $S$ that is a sectional complement of $I$ in $[(0], J]$. Otherwise, without loss of generality, we can assume that

$$
\begin{aligned}
& p \wedge s_{A}=0, \\
& p \wedge s_{B}=p .
\end{aligned}
$$

Let $s_{B}^{\prime}$ be a sectional complement of $p$ in $\left[0, s_{B}\right]$. Then $\left\langle s_{A}, s_{B}^{\prime}\right\rangle$ satisfies

$$
p \wedge s_{A}=p \wedge s_{B}^{\prime}
$$

$(=0)$, and so the pair represents an ideal $S$ of $M$. Obviously, $I \wedge S=(0]$.

Since $p \wedge s_{B}=p$, it follows that $p \leq s_{B} \leq j_{B}$. Since $J$ is an ideal and $j_{B} \wedge p=p$, it follows that $j_{A} \wedge p=p$, that is,

$$
p \leq j_{A}
$$

Obviously, $I \vee S \subseteq J$. So to show that $I \vee S=J$, it is sufficient to verify that $j_{A}$, $j_{B} \in I \vee S$. Evidently, $j_{A}=i_{A} \vee s_{A} \in I \vee S$. Note that $p \leq j_{A}=i_{A} \vee s_{A} \in I \vee S$. Thus, $p, s_{B}^{\prime}, i_{B} \in I \vee S$, and therefore

$$
p \vee s_{B}^{\prime} \vee i_{B}=\left(p \vee s_{B}^{\prime}\right) \vee i_{B}=s_{B} \vee i_{B}=j_{B} \in I \vee S,
$$

which was to be proved.

An easy consequence of this theorem is the following:

Corollary 6. Let $A, B$, and $M$ be as in Theorem 5. In addition, let us assume that $B$ is a simple lattice. Then $\operatorname{Con} M \cong \operatorname{Con} A$ and $\operatorname{Id} M$ is a sectionally complemented congruence-preserving extension of $A$.

Proof. It is clear that a congruence $\Theta$ of $A$ has exactly one extension to a congruence $\Theta_{M}$ of $M$; indeed, if $p_{A} \equiv 0_{A}(\Theta)$, then let $B$ be collapsed under $\Theta_{M}$; otherwise, let $\Theta_{M}$ be $\omega_{B}$ on $B$. So this statement follows from Theorem 5 and Lemma 4.

Lemma 7. For every finite lattice $L$, there is a finite, simple, sectionally complemented extension $S(L)$.

Proof. If $|L| \leq 2$, then we can take $S(L)=L$. So we can assume that $|L|>2$.

Let 0 and 1 be the zero and unit element of $L$, respectively. We can assume that 1 is join-reducible; otherwise, adjoin a common complement $u$, that is, an element $u$ satisfying

$$
\begin{aligned}
& u \wedge x=0, \\
& u \vee x=1,
\end{aligned}
$$


for all $x \in L-\{0,1\}$. So there are elements $x_{1}, x_{2} \in L-\{1\}$ satisfying $x_{1} \vee x_{2}=1$, and 1 is join-reducible.

Let

$$
N(L)=L-\{x \mid x=0 \text { or } x \text { is an atom }\} .
$$

For every $a \in N(L)$, we adjoin an atom $p_{a}<a$ so that if $a \neq b$, then $p_{a} \neq p_{b}$. We make

$$
S(L)=L \cup\left\{p_{a} \mid a \in N(L)\right\}
$$

(a disjoint union) into a poset by the following rules:

$(\alpha) L$ is a subposet of $S(L)$;

$(\beta)$ if $a \in N(L)$ and $x \in L$, then

$$
\begin{array}{ll}
x<p_{a} & \text { iff } x=0, \\
p_{a}<x & \text { iff } a \leq x ;
\end{array}
$$

$(\gamma)$ if $a$ and $b \in N(L)$, then

$$
p_{a} \leq p_{b} \quad \text { iff } \quad a=b .
$$

Then $S(L)$ is a lattice. The meet and the join in $S(L)$ are described by the following rules:

(i) $L$ is a sublattice of $S(L)$;

(ii) if $a \in N(L)$ and $x \in L$, then

$$
p_{a} \wedge x= \begin{cases}0, & \text { if } a \not \leq x, \\ p_{a}, & \text { if } a \leq x ;\end{cases}
$$

(iii) if $a \in N(L)$ and $x \in L$, then

$$
p_{a} \vee x= \begin{cases}a \vee x, & \text { if } x \neq 0, \\ p_{a}, & \text { if } x=0\end{cases}
$$

(iv) if $a$ and $b \in N(L), a \neq b$, then

$$
\begin{aligned}
& p_{a} \wedge p_{b}=0, \\
& p_{a} \vee p_{b}=a \vee b .
\end{aligned}
$$

Obviously, $S(L)$ is a finite lattice; 0 and 1 are the zero and unit elements of $S(L)$, respectively. $S(L)$ an extension of $L$. To show that $S(L)$ is sectionally complemented, take $0<u<v$ in $S(L)$. We consider two cases:

Case 1. $u \in L$. Then $v \in N(L)$ and $p_{v}$ is a sectional complement of $u$ in $[0, v]$ by (ii) and (iii).

Case 2. $u \notin L$. Then $u=p_{a}$, for some $a \in N(L)$, and $v \in L$ satisfies $a \leq v$. If $a=v$, then any $x$ satisfying $0<x<a$ is a sectional complement of $u$ in $[0, v]$; and there is such an $x$ because $a \in N(L)$. If $a<v$, then $p_{v}$ is a sectional complement of $u$ in $[0, v]$ by (iii) and (iv).

Finally, $S(L)$ is simple. Indeed, let $\Theta>\omega$ be a congruence of $L$. We verify that there is an $a>0, a \in S(L)$, such that $a \equiv 0(\Theta)$. Indeed, since $\Theta>\omega$, there are $u, v \in L$ such that $u<v$ and $u \equiv v(\Theta)$. If $u=0$, then $a=v$ satisfies the requirements. If $0<u$, then $v \in N(L)$, so there is an element $p_{v} \in S(L)$. We have two cases to consider: 
Case 1. $u \in L$. In this case, $u \equiv v(\Theta)$ implies that $p_{v} \equiv 0(\Theta)$, so we can take $a=p_{v}$.

Case 2. $u \in S(L)-L$. In this case, $u=p_{x}$, for some $x \in N(L)$. Obviously, $v \in L$ and $x \leq v$. Since $x \in N(L)$, there is an $a \in L$ satisfying $0<a<x$. Now $u \equiv v(\Theta)$ implies that $p_{x} \equiv x(\Theta)$ and so $a \equiv 0(\Theta)$.

Using the congruence $a \equiv 0(\Theta)$, we conclude that $p_{1} \equiv 1(\Theta)$. Meeting with $x_{1}$, we obtain that $x_{1} \equiv 0(\Theta)$, and similarly $x_{2} \equiv 0(\Theta)$. Joining these two congruences, we obtain that $0 \equiv 1(\Theta)$, that is, $\Theta=\iota$.

We should point out that since a finite partition lattice is simple and sectionally complemented by O. Ore [9], Lemma 7 follows from the following very deep result of P. Pudlák and J. Tůma [11]: Every finite lattice can be embedded into a finite partition lattice.

For the lattice $K$ of Figure 1, we can choose for $S(K)$ the lattice depicted in Figure 2; note that this is much smaller than the lattice constructed in the proof of Lemma 7.

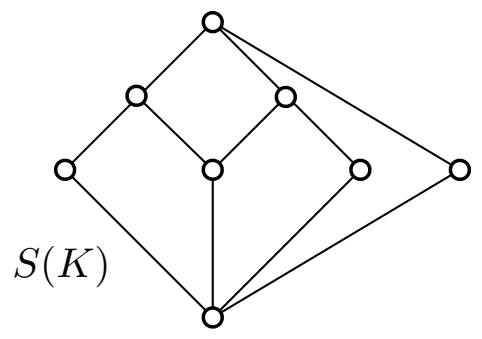

FiguRE 2

\section{Rectangular extensions}

The rectangular extension $\mathbb{R}(K)$ of a finite lattice $K$ is defined as the direct product of all subdirect factors of $K$, that is,

$$
\mathbb{R}(K)=\prod(K / \Phi \mid \Phi \in \mathrm{M}(\operatorname{Con} K)),
$$

where $\mathrm{M}(\mathrm{Con} K)$ is the set of all meet-irreducible congruences of $K$.

$K$ has a natural (diagonal) embedding into $\mathbb{R}(K)$ by

$$
\psi: a \mapsto a^{\mathbb{R}}=\langle[a] \Phi \mid \Phi \in \mathrm{M}(\operatorname{Con} K)\rangle .
$$

Let $K \psi=K^{\mathbb{R}}$ and for a congruence $\Theta$ of $K$, let $\Theta^{\mathbb{R}}$ denote the corresponding congruence of $K^{\mathbb{R}}$, that is, $\Theta^{\mathbb{R}}=\Theta \psi$.

Theorem 8. Let $K$ be a finite lattice. Then $K^{\mathbb{R}}$ has the Congruence Extension Property in $\mathbb{R}(K)$.

Proof. Let $\Theta \in \operatorname{Con} K$ and $\Phi \in \mathrm{M}(\operatorname{Con} K)$; define the congruence $\Theta_{\Phi}$ of $K / \Phi$ as follows:

$$
\Theta_{\Phi}= \begin{cases}\omega, & \text { if } \Theta \leq \Phi, \\ \iota, & \text { if } \Theta \not \leq \Phi ;\end{cases}
$$




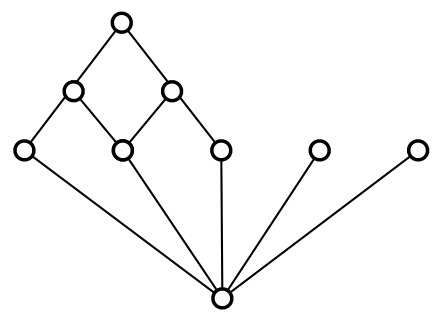

Figure 3

and define

$$
\bar{\Theta}^{\mathbb{R}}=\prod\left(\Theta_{\Phi} \mid \Phi \in \mathrm{M}(\operatorname{Con} K)\right) .
$$

Obviously, $\bar{\Theta}^{\mathbb{R}}$ is a congruence of $\mathbb{R}(K)$. It remains to show the Congruence Extension Property, that is, that

$$
u^{\mathbb{R}} \equiv v^{\mathbb{R}}\left(\Theta^{\mathbb{R}}\right) \text { in } K^{\mathbb{R}} \quad \text { iff } \quad u^{\mathbb{R}} \equiv v^{\mathbb{R}}\left(\bar{\Theta}^{\mathbb{R}}\right) \text { in } \mathbb{R}(K) \text {, for all } u, v \in K .
$$

Indeed, $u^{\mathbb{R}} \equiv v^{\mathbb{R}}\left(\Theta^{\mathbb{R}}\right)$ in $K^{\mathbb{R}}$ is equivalent to $u v(\Theta)$ in $K$, by the definition of $u^{\mathbb{R}}$, $v^{\mathbb{R}}, K^{\mathbb{R}}$, and $\Theta^{\mathbb{R}}$. The last congruence is equivalent to $u v(\Phi)$ (that is, $[u] \Phi=[v] \Phi$ ), for all $\Phi \in \mathrm{M}($ Con $K)$ satisfying $\Theta \leq \Phi$, which, in turn, can be written as

$$
[u] \Phi \equiv[v] \Phi\left(\Theta_{\Phi}\right) \text {, for all } \Phi \in \mathrm{M}(\mathrm{Con} K) \text { satisfying } \Theta \leq \Phi
$$

(since for $\Theta \leq \Phi$, by definition, $\Theta_{\Phi}=\omega$ ). Since $\Theta_{\Phi}=\iota$, for $\Theta \not \leq \Phi$, the congruence $[u] \Phi \equiv[v] \Phi\left(\Theta_{\Phi}\right)$ always holds if $\Theta \not \leq \Phi$. Therefore, the last displayed condition is equivalent to $[u] \Phi \equiv[v] \Phi\left(\Theta_{\Phi}\right)$, for all $\Phi \in \mathrm{M}(\operatorname{Con} K)$, which is the same as $u^{\mathbb{R}} \equiv v^{\mathbb{R}}\left(\Theta^{\mathbb{R}}\right)$, as claimed.

For the lattice $K$ of Figure $1, \mathbb{R}(K)$ is $K \times\left(\mathfrak{C}_{2}\right)^{2}$ (where $\mathfrak{C}_{2}$ denotes the twoelement lattice), which is representable as the ideal lattice of the chopped lattice of Figure 3. Indeed, $K$ has three meet-irreducible congruences, $\omega, \Phi_{1}$, and $\Phi_{2}$, so the three direct factors of $\mathbb{R}(K)$ are $K \cong K / \omega, K / \Phi_{1}$, and $K / \Phi_{2}$; and the latter two are isomorphic to $\mathfrak{C}_{2}$.

For each $\Phi \in \mathrm{M}(\operatorname{Con} K)$, we select $S(K / \Phi)$, a finite, simple, sectionally complemented extension of $K / \Phi$, as provided by Lemma 7 ; let $p_{\Phi}$ be an atom of $S(K / \Phi)$. Then we form the extension

$$
\widehat{\mathbb{R}}(K)=\prod(S(K / \Phi) \mid \Phi \in \mathrm{M}(\operatorname{Con} K))
$$

of $\mathbb{R}(K)$. We shall also denote by $p_{\Phi}$ the corresponding atom of $\widehat{\mathbb{R}}(K)$, that is, the element whose $\Phi$ component is $p_{\Phi}$ and all the other components are zero.

For the lattice $K$ of Figure 1, we can choose $S\left(\mathfrak{C}_{2}\right)=\mathfrak{C}_{2}$ and then $\widehat{\mathbb{R}}(K)$ can be represented as the ideal lattice of the chopped lattice of Figure 4. The three atoms singled out in the previous paragraph are black-filled in the diagram. (The first of the black-filled atoms could be any one of the first four atoms.)

Lemma 9. Let $K$ be a finite lattice. Then the extension $\widehat{\mathbb{R}}(K)$ of $K \cong K^{\mathbb{R}}$ has the following properties:

(i) $\widehat{\mathbb{R}}(K)$ is sectionally complemented;

(ii) $\widehat{\mathbb{R}}(K)$ is finite; 


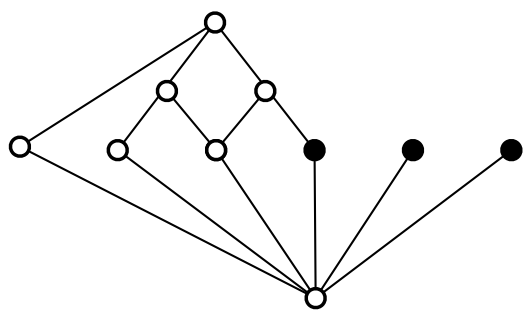

FiguRE 4

(iii) let

$$
P=\left\{p_{\Phi} \mid \Phi \in \mathrm{M}(\mathrm{Con} K)\right\}
$$

then there is a one-to-one correspondence between subsets of $P$ and congruences $\Theta$ of $\widehat{\mathbb{R}}(K)$; the subset of $P$ corresponding to the congruence $\Theta$ of $\widehat{\mathbb{R}}(K)$ is

$$
\left\{p_{\Phi} \mid p_{\Phi} \equiv 0(\Theta)\right\}
$$

hence, the congruence lattice of $\widehat{\mathbb{R}}(K)$ is a finite Boolean lattice;

(iv) every congruence $\Theta$ of $K$ has an extension $\widehat{\Theta}$ to a congruence of $\widehat{\mathbb{R}}(K)$ corresponding to the subset

$$
\left\{p_{\Phi} \mid \Phi \in \mathrm{M}(\mathrm{Con} K) \text { and } \Theta \not \leq \Phi\right\}
$$

of $P$.

Proof. Properties (i) and (ii) follow from Lemma 7. Property (iii) is trivial since $\widehat{\mathbb{R}}(K)$ is a finite direct product of simple lattices.

To verify property (iv), observe that a congruence $\Theta$ of $K$ extends to $\mathbb{R}(K)$ to a congruence that is a direct product of congruences of the form $\omega$ and $\iota$ of the factors; the corresponding direct product of congruences of the form $\omega$ and $\iota$ (on the factors $S(K / \Phi)$ ) extends $\Theta$ to a congruence $\widehat{\Theta}$ of $\widehat{\mathbb{R}}(K)$. The last statement is a rewrite of the definition of $\Theta^{\mathbb{R}}$ in the proof of Theorem 8 .

\section{Proof of the Theorem}

Let $K$ be a finite lattice. In this section, we construct a finite, sectionally complemented, congruence-preserving extension $L$ of $K$, as required by the Theorem.

Using Lemma 7, for every $\Phi \in \mathrm{M}($ Con $K)$, we select a finite, sectionally complemented, simple extension $K_{\Phi}=S(K / \Phi)$ of $K / \Phi$, with an atom, $p_{\Phi}$, and zero, $0_{\Phi}$.

Let $D=$ Con $K$, and let $L_{0}$ be a lattice whose existence was stated in Theorem $2^{\prime}$ for the finite distributive lattice $D$. Since

$$
\mathrm{J}\left(\operatorname{Con} L_{0}\right) \cong \mathrm{J}(D) \cong \mathrm{J}(\operatorname{Con} K),
$$

the independent set of atoms $Q=\left\{q_{\Phi} \mid \Phi \in \mathrm{J}\left(\operatorname{Con} L_{0}\right)\right\}$ of $L_{0}$ (described in (iv) of Theorem $2^{\prime}$ ) can also be denoted as follows:

$$
Q=\left\{q_{\Phi} \mid \Phi \in \mathrm{J}(\operatorname{Con} K)\right\},
$$

and for $\Phi, \Phi^{\prime} \in \mathrm{J}(\operatorname{Con} K), \Theta\left(0, q_{\Phi}\right) \leq \Theta\left(0, q_{\Phi^{\prime}}\right)$ iff $\Phi \leq \Phi^{\prime}$ in $\mathrm{J}(\operatorname{Con} K)$. 
For a congruence $\Theta$ of $K$, let $\Theta_{0}$ denote the congruence of $L_{0}$ determined by the set $\left\{q_{\Phi} \mid \Phi \leq \Theta\right\}$, that is,

$$
\Theta_{0}=\bigvee\left(\Theta\left(q_{\Phi}, 0_{L_{0}}\right) \mid \Phi \leq \Theta\right) ;
$$

obviously, $\Theta \mapsto \Theta_{0}$ sets up an isomorphism Con $K \rightarrow \operatorname{Con} L_{0}$.

Let $\Phi \in \mathrm{M}(\operatorname{Con} K)$; then let $\Phi^{\dagger}$ be the smallest congruence of $K$ not contained in $\Phi$. Note that $\Phi^{\dagger} \in \mathrm{J}(\mathrm{Con} K)$. The map $\Phi \mapsto \Phi^{\dagger}$ is a natural order isomorphism between $\mathrm{M}(\operatorname{Con} K)$ and $\mathrm{J}(\operatorname{Con} K)$.

The following observation is trivial but crucial:

Lemma 10. The map $p_{\Phi} \mapsto q_{\Phi^{\dagger}}$ sets up a bijection between the set

$$
\left\{p_{\Phi} \mid \Phi \in \mathrm{M}(\mathrm{Con} K) \text { and } \Theta \not \leq \Phi\right\}
$$

that describes the congruence $\widehat{\Theta}$ of $\widehat{\mathbb{R}}(K)$ in Lemma $9(\mathrm{iv})$ and the set

$$
\left\{q_{\Phi} \mid \Phi \in \mathrm{J}(\mathrm{Con} K) \text { and } \Phi \leq \Theta\right\}
$$

that describes the congruence $\Theta_{0}$ in $L_{0}$.

In addition,

$$
\left\{q_{\Phi} \mid \Phi \in \mathrm{J}(\operatorname{Con} K) \text { and } \Phi \leq \Theta\right\}=\left\{q_{\Phi^{\dagger}} \mid \Phi \in \mathrm{M}(\operatorname{Con} K) \text { and } \Theta \not \leq \Phi\right\} .
$$

Now we inductively construct the lattice $L$ of the Theorem. Let

$$
\Phi_{1}, \Phi_{2}, \ldots, \Phi_{n}
$$

list the meet-irreducible congruences of $K$. We apply Theorem 5 with $A=L_{0}$, $B=S\left(K / \Phi_{1}\right)$, and the atoms $q_{\Phi_{1}^{\dagger}}$ of $L_{0}$ and $p_{\Phi_{1}}$ of $S\left(K / \Phi_{1}\right)$, to obtain the chopped lattice $M_{1}$ and $L_{1}=\operatorname{Id} M_{1}$. From Corollary 6 , it follows that $L_{1}$ is a congruencepreserving extension of $L_{0}$ and that $L_{1}$ also satisfies property (iv) of Theorem $2^{\prime}$, in fact, with the same set of atoms as for $L_{0}$. Moreover, $L_{1}$ is finite and sectionally complemented by Theorem 5 . So we can apply Theorem 5 with $A=L_{1}, B=$ $S\left(K / \Phi_{2}\right)$, and the atoms $q_{\Phi_{2}^{\dagger}}$ of $L_{1}$ and $p_{\Phi_{2}}$ of $S\left(K / \Phi_{2}\right)$, obtaining the chopped lattice $M_{2}$ and $L_{2}=\operatorname{Id} M_{2}$. In $n$ steps, we construct $M_{n}$ and $L=L_{n}=\operatorname{Id} M_{n}$.

It is clear that $L$ is a finite sectionally complemented lattice. It is also evident that $L$ is a congruence-preserving extension of $L_{0}$, hence, Con $L \cong D$. So to complete the proof of the Theorem, we have to show that $K$ has an embedding into $L$ with the Congruence Extension Property.

In the next step, we need the following statement:

Lemma 11. Let $A, B, C$ be pairwise disjoint finite lattices with zeroes $0_{A}, 0_{B}, 0_{C}$, and atoms $p_{A} \in A, p_{B}, p_{B}^{\prime} \in B, p_{B} \neq p_{B}^{\prime}, p_{C} \in C$. We construct some chopped lattices.

(i) Let $N_{1}$ be the chopped lattice obtained by forming the disjoint union of $A$ and $B$ and identifying $0_{A}$ with $0_{B}$ and $p_{A}$ with $p_{B}$.

(ii) Let $N$ be the chopped lattice obtained by forming the disjoint union of $A, B$, $C$ and identifying $0_{A}$ with $0_{B}$ and $0_{C}, p_{A}$ with $p_{B}$, and $p_{B}^{\prime}$ with $p_{C}$.

(iii) Form the ideal lattice Id $N_{1}$, which we consider an extension of $N_{1}$, so it has atoms $p_{A}=p_{B}$ and $p_{B}^{\prime}$ and let $N_{2}$ be the chopped lattice that is the disjoint union of $\operatorname{Id} N_{1}$ and $C$ with the zeroes identified and also the atom $p_{B}^{\prime} \in \operatorname{Id} N_{1}$ is identified with the atom $p_{C} \in C$.

Then $\operatorname{Id} N_{2}$ is isomorphic to $\operatorname{Id} N$. 
Proof. The elements of Id $N_{1}$ are pairs $\langle a, b\rangle \in A \times B$ satisfying $a \wedge p_{a}=b \wedge p_{b}$. So the elements of Id $N_{2}$ are pairs $\langle\langle a, b\rangle, c\rangle$ such that $\langle a, b\rangle \in \operatorname{Id} N_{1}$ and $\langle a, b\rangle \wedge p_{B}^{\prime}=c \wedge p_{C}$; note that $\langle a, b\rangle \in \operatorname{Id} N_{1}$ iff $a \wedge p_{A}=b \wedge p_{B}$ and note also that $\langle a, b\rangle \wedge p_{B}^{\prime}=b \wedge p_{B}^{\prime}$. On the other hand, the elements of $\operatorname{Id} N$ are triples $\langle a, b, c\rangle \in A \times B \times C$ satisfying $a \wedge p_{A}=b \wedge p_{B}$ and $b \wedge p_{B}^{\prime}=c \wedge p_{C}$. It should now be obvious that $\langle\langle a, b\rangle, c\rangle \mapsto\langle a, b, c\rangle$ is the required isomorphism Id $N_{2} \rightarrow \operatorname{Id} N$.

Another view of $L$ is the following. We form the chopped lattice $M_{1}$; instead of proceeding to $L_{1}=\operatorname{Id} M_{1}$, let us now form the chopped lattice $M_{2}^{\prime}$ from $M_{1}=M_{1}^{\prime}$ and $S\left(K / \Phi_{2}\right)$ by identifying the zeros of $M_{1}^{\prime}$ and $S\left(K / \Phi_{2}\right)$ and the atom $q_{\Phi_{2}^{\dagger}}$ of $M_{1}^{\prime}$ with the atom $p_{\Phi_{2}}$ of $S\left(K / \Phi_{2}\right)$. Observe that $M_{2}^{\prime}$ is the union of $L_{0}, S\left(K / \Phi_{1}\right)$, and $S\left(K / \Phi_{2}\right)$ and $S\left(K / \Phi_{1}\right) \cap S\left(K / \Phi_{2}\right)=\{0\}$. By Lemma 11, Id $M_{2}^{\prime} \cong L_{2}$. Proceeding thus, we obtain the chopped lattice

$$
M_{n}^{\prime}=L_{0} \cup S\left(K / \Phi_{1}\right) \cup S\left(K / \Phi_{2}\right) \cup \cdots \cup S\left(K / \Phi_{n}\right),
$$

where, for all $1 \leq i<j \leq n$, we have $S\left(K / \Phi_{i}\right) \cap S\left(K / \Phi_{j}\right)=\{0\}$, and $L \cong \operatorname{Id} M_{n}^{\prime}$, again by Lemma 11. So the chopped sublattice $S\left(K / \Phi_{1}\right) \cup S\left(K / \Phi_{2}\right) \cup \cdots \cup S\left(K / \Phi_{n}\right)$ of $M_{n}^{\prime}$ is just the lattices $S\left(K / \Phi_{1}\right), S\left(K / \Phi_{2}\right), \ldots, S\left(K / \Phi_{n}\right)$ glued together at their zeros, and, therefore,

$$
\operatorname{Id}\left(S\left(K / \Phi_{1}\right) \cup S\left(K / \Phi_{2}\right) \cup \cdots \cup S\left(K / \Phi_{n}\right)\right) \cong \widehat{\mathbb{R}}(K) .
$$

Thus we can view $L$ as the ideal lattice of the chopped lattice obtained by gluing together $L_{0}$ and $\widehat{\mathbb{R}}(K)$ over the Boolean lattice of $2^{n}$ elements generated in $L_{0}$ and in $\widehat{\mathbb{R}}(K)$ by $P=Q$. A congruence $\Theta$ of $L$ is then determined by a congruence $\Theta_{L_{0}}$ on $L_{0}$ and a congruence $\Theta_{\widehat{\mathbb{R}}(K)}$ on $\widehat{\mathbb{R}}(K)$ that agree on the Boolean lattice. Since $\widehat{\mathbb{R}}(K)$ is a Congruence Preserving Extension of $K \cong K^{\mathbb{R}}$, it follows now that $L$ is a Congruence Preserving Extension of $K$, concluding the proof of the Theorem.

Figure 5 shows the chopped lattice whose ideal lattice is the lattice $L$ (more precisely, one candidate for the lattice $L$ ) of the Theorem for the lattice $K$ of Figure 1. As we pointed out in connection with Figure 3, the chopped lattice whose ideal lattice is $\widehat{\mathbb{R}}(K)$ consists of $S(K)$ and two more atoms, which are incorporated in $L_{0}$. So in this very easy illustration, the chopped lattice whose ideal lattice is $L$, in fact, consists of $S(K)$ and $L_{0}$ identified by their zeros and one atom each. $L_{0}$ is also the smallest nontrivial example of the Theorem. It is easy to compute that $L$ has 40 elements.

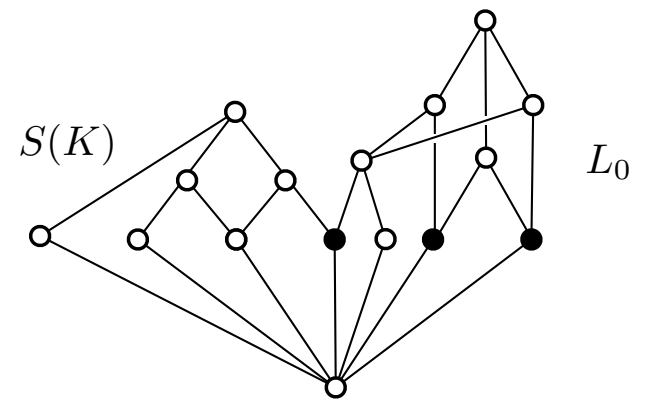

Figure 5 


\section{ADDENDUM}

While revising this paper (Aug. 1997), we were working on a problem proposed in our paper with H. Lakser [5] and we realized that generalizing the Theorem in this paper would yield a solution (see below).

Here is the generalization:

Theorem'. Let $K$ be a lattice and let $\Gamma$ be a finite $\{0,1\}$-meet subsemilattice of the congruence lattice of $K$. If $\Gamma$ is a distributive lattice, then $K$ has an extension $L$ satisfying the following properties:

(i) L is sectionally complemented;

(ii) the congruence lattice of $L$ is isomorphic to $\Gamma$;

(iii) a congruence $\Phi$ of $K$ has an extension to $L$ iff $\Phi \in \Gamma$;

(iv) each $\Phi \in \Gamma$ has a unique extension to $L$.

We present two consequences of Theorem'.

Corollary 12. Every lattice $K$ with a finite congruence lattice has a congruencepreserving embedding into a sectionally complemented lattice $L$.

Indeed, if Con $K$ is finite, then set $\Gamma=$ Con $K$ and apply Theorem'.

In the Introduction, we mention the problem whether every countable lattice has a congruence-preserving extension to a sectionally complemented lattice. In light of Theorem', we would like to rephrase this:

Problem. Let $K$ be a lattice with the property that $K$ has countably many compact congruence relations. Is it true that $K$ has a congruence-preserving extension to a sectionally complemented lattice?

Corollary 13. Let $K$ be a lattice and let $\Phi$ be a nontrivial congruence of $K$. Then there is an extension $L$ of $K$ and a standard ideal $S$ of $L$ such that the restriction of $\Theta[S]$ to $K$ is $\Phi$. Moreover, $L$ is sectionally complemented and it has only one nontrivial congruence, namely, $\Theta[S]$.

This is a much stronger form of a result in the paper [5] by H. Lakser and the authors, in which it is proved that there is an extension $L$ of $K$ and a standard ideal $S$ of $L$ such that the restriction of $\Theta[S]$ (the smallest congruence under which $S$ is in a single congruence class) to $K$ is $\Phi$ but the additional properties of $L$ stated in the last sentence of Corollary 13 do not hold in the former construction.

We get Corollary 13 from Theorem', by setting $\Gamma=\{\omega, \Phi, \iota\}$.

We raised the problem in [5] whether the result in that paper can be generalized to finitely many congruences; the construction in [5] cannot be used to prove such a result; for instance, if we apply the construction twice, the standard ideal obtained in the first step is no longer standard after the second step. Of course, Theorem' gives a positive solution to this problem.

Changes in the proof. In conclusion, we describe the changes necessary in the proof of the Theorem, to obtain a proof of Theorem'.

The crucial step is the modification of Lemma 4; this was done in [7]. Let us say that a chopped lattice $M$ satisfies (FG), if every finitely generated ideal is a finite union of principal ideals. If $M$ satisfies (FG), then $\operatorname{Id}_{\mathrm{fg}} M$ (the poset of finitely generated ideals of $M$ ) forms a sublattice of Id $M$. 
Lemma $4^{\prime}$. Let $M$ be a chopped lattice satisfying (FG). Then the lattice $\operatorname{Id}_{\mathrm{fg}} M$ is a congruence-preserving extension of the chopped lattice $M$.

This form of Lemma 4 should be used throughout the new proof. Note that all chopped lattices in the proof satisfy (FG).

To generalize Theorem 5 to arbitrary lattices, replace $\operatorname{Id} M$ by $\operatorname{Id}_{\mathrm{fg}} M$.

The rectangular extension $\mathbb{R}(K)$ of a finite lattice $K$ has to be changed to a $\Gamma$-rectangular extension of an arbitrary lattice $K$ as follows:

$$
\mathbb{R}_{\Gamma}(K)=\prod(K / \Phi \mid \Phi \in \mathrm{M}(\Gamma)) ;
$$

and we define

$$
\widehat{\mathbb{R}}_{\Gamma}(K)=\prod(S(K / \Phi) \mid \Phi \in \mathrm{M}(\Gamma),
$$

where $S(K / \Phi)$ is a simple, sectionally complemented extension of $K / \Phi$ with an atom $p_{\Phi}$.

Lemma 9 remains true, except that the finiteness statement has to be dropped.

In Section 5 , we have to make very few changes. To start, define $D=\Gamma$ (this step utilizes that $\Gamma$ is distributive), and replace all references to Con $K$ by $\Gamma$, so, for instance,

$$
Q=\left\{q_{\Phi} \mid \Phi \in \mathrm{J}(\Gamma)\right\}
$$

Now we inductively construct the lattice $L$, except that

$$
\Phi_{1}, \Phi_{2}, \ldots, \Phi_{n}
$$

lists the meet-irreducible congruences of $\Gamma$. Lemma 11 is valid with the only change that we form $\operatorname{Id}_{\text {fg }} N, \operatorname{Id}_{\text {fg }} N_{1}$, and $\operatorname{Id}_{\text {fg }} N_{2}\left(\right.$ rather than $\operatorname{Id} N, \operatorname{Id} N_{1}$, and $\left.\operatorname{Id} N_{2}\right)$ ).

\section{REFERENCES}

[1] G. Grätzer, General Lattice Theory, Pure and Applied Mathematics 75, Academic Press, Inc. (Harcourt Brace Jovanovich, Publishers), New York-London; Lehrbücher und Monographien aus dem Gebiete der Exakten Wissenschaften, Mathematische Reihe, Band 52. Birkhäuser Verlag, Basel-Stuttgart; Akademie Verlag, Berlin, 1978. xiii+381 pp. (Expanded Second Edition, 1998.) MR 80c:06001b

[2] G. Grätzer, H. Lakser, and E. T. Schmidt, Isotone maps as maps of congruences. I. Abstract maps, Acta Math. Acad. Sci. Hungar. 75 (1997), 81-111. CMP 97:10

[3] _ Representing isotone maps as maps of congruences. II. Concrete maps, manuscript.

[4] - Congruence representations of join homomorphisms of distributive lattices: A short proof, Math. Slovaca 46 (1996), 363-369. CMP 98:02

[5] Restriction of standard congruences on lattices, manuscript. Accepted for publication in Contributions to General Algebra 10, Proceedings of the Klagenfurt Conference May 29 June 1, 1997. Edited by D. Dorninger, E. Eigenthaler, H. J. Kaiser, H. Kautschitsch, W. More and W. B. Müller. B. G. Teubner, Stuttgart. Aug. 1997.

[6] G. Grätzer and E. T. Schmidt, On congruence lattices of lattices, Acta Math. Acad. Sci. Hungar. 13 (1962), 179-185. MR 25:2983

[7] _ A lattice construction and congruence-preserving extensions, Acta Math. Hungar. 66 (1995), 275-288. MR 95m:06018

[8] _ The Strong Independence Theorem for automorphism groups and congruence lattices of finite lattices, Beiträge Algebra Geom. 36 (1995), 97-108. MR 96h:06014

[9] O. Ore, Theory of equivalence relations, Duke Math. J. 9 (1942), 573-627. MR 4:128f

[10] M. Ploščica, J. Tůma, and F. Wehrung, Congruence lattices of free lattices in non-distributive varieties, Colloq. Math. 76 (1998), 269-278. 
[11] P. Pudlák and J. Tůma, Every finite lattice can be embedded into a finite partition lattice, Algebra Universalis 10 (1980), 74-95. MR 81e:06013

[12] M. Tischendorf, The representation problem for algebraic distributive lattices, $\mathrm{Ph}$. D. thesis, Fachbereich Mathematik der Technischen Hochschule Darmstadt, Darmstadt, 1992. MR 95g:06010

Department of Mathematics, University of Manitoba, Winnipeg, Manitoba, Canada R3T $2 \mathrm{~N} 2$

E-mail address: gratzer@cc.umanitoba.ca

Mathematical Institute of the Technical University of Budapest, Müegyetem RKP. 3, H-1521 Budapest, Hungary

E-mail address: schmidt@math.bme.hu 